\title{
Using a small-core graphite calorimeter for dosimetry and scintillator quenching corrections in a therapeutic proton beam
}

Christensen, Jeppe Brage; Vestergaard, Anne; Andersen, Claus E.

Published in:

Physics in Medicine and Biology

Link to article, DOI:

$10.1088 / 1361-6560 / a b 9 b c 3$

Publication date:

2020

Document Version

Peer reviewed version

Link back to DTU Orbit

Citation (APA):

Christensen, J. B., Vestergaard, A., \& Andersen, C. E. (2020). Using a small-core graphite calorimeter for dosimetry and scintillator quenching corrections in a therapeutic proton beam. Physics in Medicine and Biology, 65(21), [215023]. https://doi.org/10.1088/1361-6560/ab9bc3

\section{General rights}

Copyright and moral rights for the publications made accessible in the public portal are retained by the authors and/or other copyright owners and it is a condition of accessing publications that users recognise and abide by the legal requirements associated with these rights.

- Users may download and print one copy of any publication from the public portal for the purpose of private study or research.

- You may not further distribute the material or use it for any profit-making activity or commercial gain

- You may freely distribute the URL identifying the publication in the public portal 


\section{ACCEPTED MANUSCRIPT}

\section{Using a small-core graphite calorimeter for dosimetry and scintillator quenching corrections in a therapeutic proton beam}

To cite this article before publication: Jeppe Brage Christensen et al 2020 Phys. Med. Biol. in press https://doi.org/10.1088/1361-6560/ab9bc3

\section{Manuscript version: Accepted Manuscript}

Accepted Manuscript is "the version of the article accepted for publication including all changes made as a result of the peer review process, and which may also include the addition to the article by IOP Publishing of a header, an article ID, a cover sheet and/or an 'Accepted

Manuscript' watermark, but excluding any other editing, typesetting or other changes made by IOP Publishing and/or its licensors"

This Accepted Manuscript is $\odot 2020$ Institute of Physics and Engineering in Medicine.

During the embargo period (the 12 month period from the publication of the Version of Record of this article), the Accepted Manuscript is fully protected by copyright and cannot be reused or reposted elsewhere.

As the Version of Record of this article is going to be / has been published on a subscription basis, this Accepted Manuscript is available for reuse under a CC BY-NC-ND 3.0 licence after the 12 month embargo period.

After the embargo period, everyone is permitted to use copy and redistribute this article for non-commercial purposes only, provided that they adhere to all the terms of the licence https://creativecommons.org/licences/by-nc-nd/3.0

Although reasonable endeavours have been taken to obtain all necessary permissions from third parties to include their copyrighted content within this article, their full citation and copyright line may not be present in this Accepted Manuscript version. Before using any content from this article, please refer to the Version of Record on IOPscience once published for full citation and copyright details, as permissions will likely be required. All third party content is fully copyright protected, unless specifically stated otherwise in the figure caption in the Version of Record.

View the article online for updates and enhancements. 

averaged over the volume of the graphite core can therefore be deduced as a temperature change

\footnotetext{
${ }^{1}$ Department of Health Technology, Technical University of Denmark, Roskilde, Denmark ${ }^{2}$ Department of Radiation Safety and Security, Paul Scherrer Institut, Villigen PSI, Switzerland ${ }^{3}$ Danish Centre for Particle Therapy, Aarhus University Hospital, Aarhus, Denmark
}

May 29, 2020

\begin{abstract}
Organic plastic scintillation detectors (PSDs) are known to produce less light per absorbed dose
\end{abstract} in highly dense radiations in comparison with e.g. ${ }^{60} \mathrm{Co}$ gamma beams. This so-called ionization density quenching can be experimentally determined by comparison of the scintillator output with the absorbed dose established with a reference detector. The hypothesis of this work was that a newly developed small-core graphite calorimeter (core size: $\varnothing 5 \mathrm{~mm} \times 7 \mathrm{~mm}$ ) can be used as reference for such measurements. The potential benefit of a calorimetric reference would be to have a robust and accurate reference with well-understood dosimetry properties even in high-intensity FLASH beams. As a first step, the hypothesis was tested by comparing previously established quenching parameter estimates for the BCF- 60 scintillating material with data obtained with the new instrument at different depths along the central axis of a $170 \mathrm{MeV}$ scanned proton beam. After the calorimetric measurements, scintillator measurements were acquired under equivalent conditions by positioning the PSD in a replica graphite core nominally identical to the core used for calorimetry. To experimentally document details of the irradiations, the spot width was mapped along the central beam axis using a new technique based on a PSD and a time-to-distance conversion procedure. Analysing the proton data in the framework of the Birks model, the graphite calorimeter gave a $k B=(11.2 \pm 0.4) \mathrm{mg} \mathrm{MeV}^{-1} \mathrm{~cm}^{-2}$ quenching parameter for BCF-60 in agreement with literature values. The consistency between the calorimetric results and the other sources of information supports the validity of the new method, and we therefore aim to apply it for characterization of other detectors in more intense beams where ionometry cannot serve as reference.

\section{Introduction}

Calorimeters can be used for absolute dosimetry in beams of ionizing radiation, and most of the primary dosimetry standards worldwide are based on water or graphite calorimetry (McEwen and Duane, 2000; Duane et al., 2012; Pinto et al., 2016; Lye et al., 2016; Renaud et al., 2018). The key feature of graphite calorimeters is that the signal production is relatively well understood such that it can be assumed that the imparted energy is solely converted to heat with no influence of chemical changes or other processes in the irradiated medium. Under adiabatic conditions, the absorbed dose
Using a small-core graphite calorimeter for dosimetry and scintillator quenching corrections in a therapeutic proton beam *E-mail: jeppe.christensen@psi.ch 
multiplied by the specific heat capacity of graphite. The main challenges in graphite calorimetry are (i) to correct for imperfect adiabatic conditions, (ii) to correct for volume averaging and scatter conditions, especially in small, non-uniform beams, and (iii) to correct from dose-to-graphite to dose-to-water. There are many solutions to these and additional technical problems as discussed in a recent comprehensive review (Renaud et al., 2020).

In comparison with calorimeters, solid-state dosimetry systems based on optical (Kry et al., 2020), electrical (Marsolat et al., 2016) or other signals (Waldeland et al., 2010) generally have less ideal (or unknown) signal production properties. In particular, organic plastic scintillation detectors (PSDs) are known to produce less light per absorbed dose in highly dense radiations in comparison with e.g. ${ }^{60}$ Co gamma beams (Torrisi, 2000; Archambault et al., 2008; Wang et al., 2012; Santurio and Andersen, 2019; Santurio et al., 2020). Although these detectors have a high degree of water equivalence with respect to how energy is imparted into the medium, they have issues with the signal production even within specific medical applications. In contrast, gas filled ionization chambers have a near constant $W$-value over a wide range of conditions (Burns, 2012), but for applications such as small-field dosimetry in MV photon beams, they require large corrections due to the perturbation caused by the air cavity, i.e. they lack water equivalence in the absence of Fano conditions (Kumar et al., 2015). For applications in FLASH intense beams (yan de Water et al., 2019), ionization chambers suffer from significant corrections due to initial and general recombination losses (Christensen et al., 2020).

The motivation for the calorimeter system discussed here, was therefore to establish a platform for experimental studies of changes of the signal production per absorbed dose for different less ideal detectors for use in new radiotherapy modalities. The hypothesis was that a newly developed small-core graphite calorimeter (Christensen et al., 2020) can be used as reference for such measurements. As indicated above, the potential benefit of a calorimetric reference would be to have a robust and accurate reference with well-understood dosimetry properties even in high-intensity beams. With some inspiration from the megavoltage calorimetric system developed by the BIPM (Picard et al., 2009), the key idea was to have a graphite core of a similar size as the detector to be characterized, and to perform irradiations of the calorimeter and the detector to be characterized under very similar scatter conditions.

The main purpose of the work presented here was to demonstrate the method with an assessment of the so-called ionization density quenching for a plastic scintillation detector at different depths along the central axis of a $170 \mathrm{MeV}$ scanned proton beam. All irradiations were carried out at dose rates comparable to what is currently used clinically $\left(<10 \mathrm{~Gy} \mathrm{~s}^{-1}\right)$. The imperfections in the adiabatic conditions underpinning the calorimetric measurements were investigated with heat-flow modelling and by analysing the experimental pre- and post-irradiation temperature traces. Monte Carlo modelling was used to account for differences between the calorimeter and the scintillator 72 detector with respect to absorption characteristics and signal averaging. Another potential challenge is the use of a spot-scanning system, which generally causes a non-uniform heating of the graphite core, and a complex heat transfer loss. The fibre-coupled PSD used for quenching investigations 
was used to measure the width of the proton spots inside a graphite core replica. Information about the graphite core, is relevant for detailed simulations of the heat transfer in the calorimeter.

\section{Materials and Methods}

The absorbed dose to the graphite core $D_{\text {core }}$ with specific heat capacity $c_{p \text {,core }}$ is related to the temperature change $\Delta T_{\text {core }}$ in a quasi-adiabatic mode as

$$
D_{\text {core }}=c_{p, \text { core }} \cdot \Delta T_{\text {core }} \cdot \prod_{i} k_{i},
$$

where $k_{i}$ denotes the correction factors, as the one for heat transfer during the irradiation $k_{\mathrm{ht}}$. The heat transfer correction factor is assessed by means of simulations and measurements as outlined below.

This study demonstrates the use of the graphite calorimeter to correct the ionization quenching in a plastic scintillator along the central axis of a scanning proton beam. Thus, the value of the specific heat capacity $c_{p \text {,core }}$ is not needed for this work, but only that the specific heat capacity remains constant throughout the experiment and that it is independent of the proton beam.

\subsection{Calorimeter design}

The calorimeter is sketched in figure 1 and described in detail in Christensen et al. (2020). A $30 \mathrm{~cm} \times$ $30 \mathrm{~cm} \times 36 \mathrm{~cm}$ PMMA phantom contains the calorimeter components in a near-vacuum situation with typical air pressures of the order of $1 \mathrm{~Pa}$. An important element is the hollow $10 \mathrm{~mm}$ thick solid water slab (Solid Water, Model 457-CFG, Gammex, USA) as outlined in figure 1. The solid water slab can accommodate one of the following:

4 ( $\left.A_{1}\right)$ A $\varnothing 5 \mathrm{~mm} \times 7 \mathrm{~mm}$ graphite core connected to a Wheatstone-bridge for temperature measurements.

$\left(A_{2}\right)$ A graphite replica of the core. The replica has a hole matching the $1 \mathrm{~mm}$ outer diameter of the PSD drilled through its centre.

The active graphite core is removable and can be replaced by the replica core containing the PSD retrofitted for a scintillator measurement in the same geometry with a nominal identical particle spectrum. This configuration allows the PSD response to be corrected for ionization quenching relative to the graphite response.

A second PSD was placed at the front of the entrance window as depicted in figure 1 to measure the beam delivery and spot sizes. Both $\varnothing 0.5 \mathrm{~mm} \times 2 \mathrm{~mm}$ polystyrene PSDs (BCF-60, SaintGobain, France) were attached to $15 \mathrm{~m}$ long optical fiber cables that in turn were connected to the photomultiplier-tubes in a ME40 instrument developed by the Technical University of Denmark 
(Beierholm et al., 2011, 2014). To resolve all individual beam spots, the ME40 was set to acquire data with a $1 \mathrm{kHz}$ sampling rate.

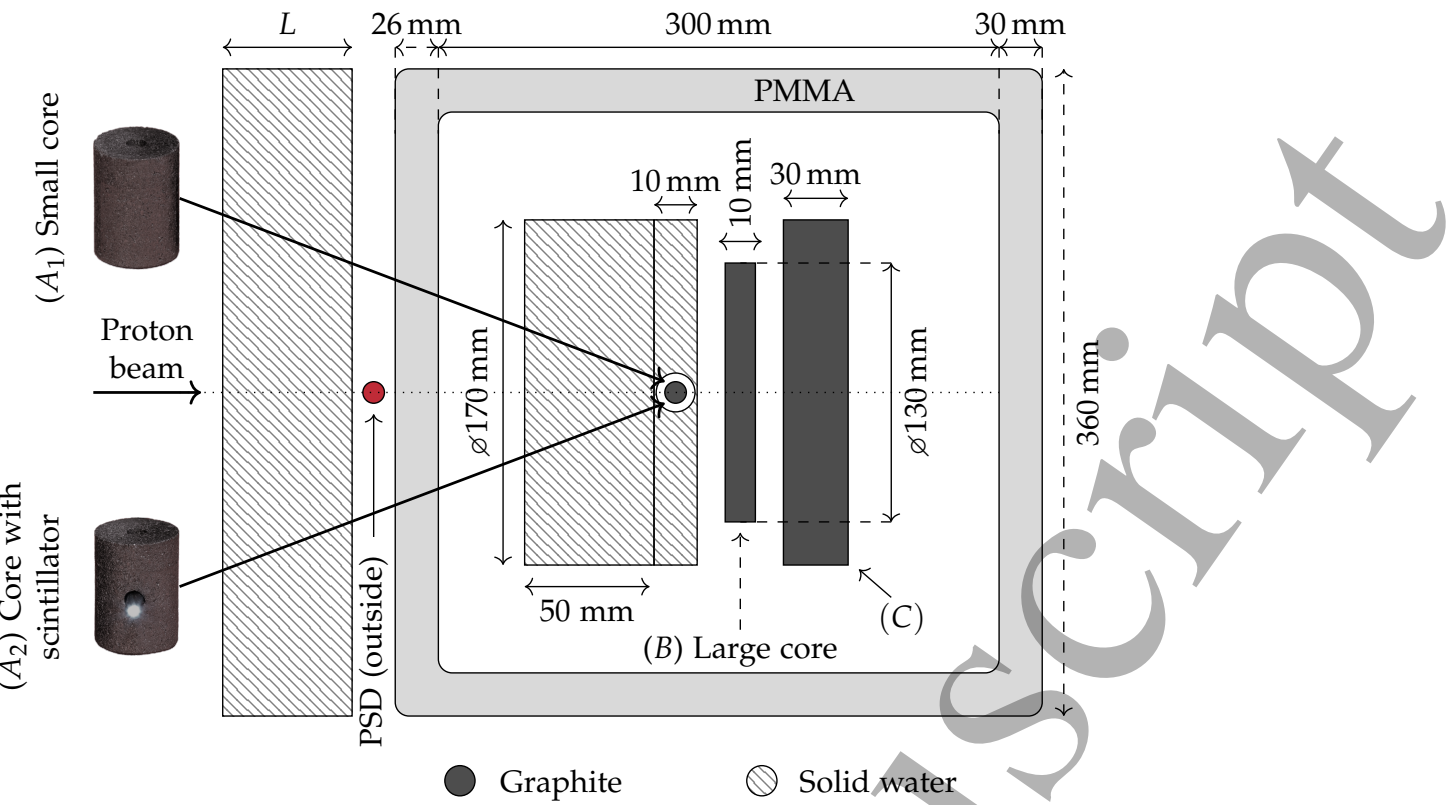

Figure 1: The calorimeter consists of a small $(A)$ and larger graphite core $(B)$ contained in a PMMA phantom connected to a vacuum pump. The smaller graphite core $A$ can either be a core used for temperature measurements $\left(A_{1}\right)$ or a replica accommodating a PSD used for quenching measurements $\left(A_{2}\right)$. A PSD is placed outside the PMMA phantom to monitor the delivery of the spotscanning field. Figure not to scale.

A larger graphite disc $(\varnothing 130 \mathrm{~mm} \times 10 \mathrm{~mm}$, denoted $B)$ was placed downstream and connected to another Wheatstone-bridge for independent temperature measurements. The results from this larger core provided dose-area-product measurements, but these results are not discussed here. A $\varnothing 170 \mathrm{~mm} \times 30 \mathrm{~mm}$ graphite disc (denoted with a $C$ ) at the distal part of the PMMA container served to minimize the radiative heat transfer from the large active graphite disc.

The calorimeter thus combines the use of graphite cores for absolute dosimetry with PSDs having a sub-mm spatial resolution and millisecond temporal resolution for improved dosimetry, where the energy and temporal deposition of each beam spot is accounted for.

\subsubsection{Thermometry}

The temperature change $\Delta T_{\text {core }}$ was measured with a Wheatstone resistance bridge and a small bead thermistor (Sensor Scientific, New Jersey, USA; model S14A10325 $\varnothing 360 \mu \mathrm{m}$ bead; $100 \mathrm{~mm}$ long $\varnothing 28 \mu \mathrm{m}$ lead wires) embedded in the graphite core. The bridge was driven by a Keithley 6121 current source and the off-balance bridge voltage was measured with a Keithley 2182A nanovoltmeter. The Keithley instruments were run in delta mode with a current constantly at $20 \mu \mathrm{A}$, a reversed polarity every $100 \mathrm{~ms}$, and voltage readings starting $1.7 \mathrm{~ms}$ after each reversal. For a balanced bridge, half of the stated current will go through the thermistor, supplying a constant power of about $1 \mu \mathrm{W}$ to the graphite core. The system was found to be highly linear and stable, and capable of measuring 
a $0.1 \Omega$ change on a $10 \mathrm{k} \Omega$ resistor with a reproducibility of $0.3 \%(k=1)$. The full system was calibrated in situ for thermometry by comparison of the bridge voltage output and with traceable reference temperature measurements.

\subsection{Experimental setup}

The irradiations of the calorimeter were undertaken at the Danish Centre for Particle Therapy (DCPT) in Aarhus, Denmark. The $2 \mathrm{~cm} \times 2 \mathrm{~cm}$ unmodulated $170 \mathrm{MeV}$ proton field consisted of $5 \times 5$ spots with a dose deposition about $2 \mathrm{~Gy}$ at the entrance region in about $0.9 \mathrm{~s}$. The position of the Bragg peak in the graphite calorimeter was varied with different thicknesses $L$ (as defined in figure 1) of solid water slabs in front of the PMMA phantom. Each irradiation was repeated 5 times. The measurement series with the graphite core was subsequently repeated, where the small graphite core is replaced with the PSD-containing graphite replica to measure the PSD response inside the graphite replica for quenching corrections and spot size measurements.

\subsection{Simulations}

\subsubsection{Monte Carlo simulations}

The spot-scanning field and a model of the calorimeter as sketched in figure 1 was implemented in Geant4 with simulation parameters given in table 1. The use of the Monte Carlo model is threefold:

(i) to score the fluence-averaged linear energy transfer $\left(\mathrm{LET}_{\Phi}\right)$ in the PSD for the ionization quenching correction. The $\mathrm{LET}_{\Phi}$ is scored following the recommendations of Cortés-Giraldo and Carabe (2015), where the $\mathrm{LET}_{\Phi}$ for a given depth is averaged over all 25 pencil beams constituting the $2 \mathrm{~cm} \times 2 \mathrm{~cm}$ field.

(ii) to investigate the signal averaging measured with the PSD and graphite core along the central beam axis. The different sizes and densities of the PSD and the graphite core give rise to different responses, here collectively denoted as volume averaging. The volume averaging correction factor is computed with the Monte Carlo model as

$$
k_{\mathrm{vol}}^{\mathrm{MC}}=\frac{D_{\mathrm{PSD}}^{\mathrm{MC}}}{D_{\mathrm{core}}^{\mathrm{MC}}}
$$

where $D_{\text {core }}^{\mathrm{MC}}$ is the dose to the graphite core and $D_{\mathrm{PSD}}^{\mathrm{MC}}$ is the dose scored in the PSD. $k_{\mathrm{vol}}^{\mathrm{MC}}$ will thus be approximately constant at the plateau region of the proton beam and gradually increase towards the Bragg peak as the graphite core is both denser and larger.

(iii) to compare the dose deposited in the graphite core $\left(D_{\text {core }}^{\mathrm{MC}}\right)$ to the measured temperate change of the core $\left(\Delta T_{\text {core }}\right)$ along the central beam axis which gives information about the heat loss during 156 the irradiation. 
Table 1: Summary of the Monte Carlo simulation parameters as recommended by the AAPM TG268 (Sechopoulos et al., 2017).

\begin{tabular}{|c|c|c|}
\hline Item name & Description & References \\
\hline Code, version & Geant4 v.10.3 (patch 03) & $\begin{array}{l}\text { Agostinelli et } \\
\text { (2003) }\end{array}$ \\
\hline Validation & $\begin{array}{l}\text { Beam characterizations }{ }^{a} \text {. Agreement with the mea- } \\
\text { surements in figure } 5\end{array}$ & \\
\hline Source description & $\begin{array}{l}2 \mathrm{~cm} \times 2 \mathrm{~cm} 170 \mathrm{MeV} \text { proton field of } 5 \times 5 \text { spots, } \\
\text { each with } \sigma_{x}=\sigma_{y}=3.96 \mathrm{~mm} \text { in air. }\end{array}$ & \\
\hline Transport parameters & $\begin{array}{l}\text { The default Geant4_Modular physics list as defined } \\
\text { in the TOPAS docs }\end{array}$ & 012) \\
\hline $\begin{array}{l}\text { Variance reduction } \\
\text { technique (VRT) }\end{array}$ & No VRT was applied & \\
\hline Scored quantities & $\begin{array}{l}\text { Dose to the PSD and dose to the graphite core. } \\
\mathrm{LET}_{\Phi} \text { in the PSD }\end{array}$ & $\begin{array}{l}\text { Cortés-Giraldo and } \\
\text { Carabe (2015) }\end{array}$ \\
\hline \# histories & $\begin{array}{l}10^{9} \text { primaries for each simulation: }<0.1 \% \text { statisti- } \\
\text { cal uncertainty }\end{array}$ & \\
\hline Statistical methods & $\begin{array}{l}\text { Type B uncertainty of } k_{\mathrm{vol}} \text { and } \mathrm{LET}_{\Phi} \text { estimated at } \\
\text { the Bragg peak for a } 1 \mathrm{~mm} \text { offset }\end{array}$ & \\
\hline Postprocessing & $\begin{array}{l}k_{\text {vol }} \text { correction based on the dose scoring, eq. (2). } \\
\text { LET }_{\Phi} \text { used for quenching corrections }\end{array}$ & \\
\hline
\end{tabular}

${ }^{a}$ Unpublished MSc Thesis.

\subsubsection{Heat transfer simulations}

A dose $D_{\text {core }}$ deposited to the graphite core will, if all energy is expressed as heat, give a temperature rise $\Delta T_{\text {core }}=D_{\text {core }} / c_{p, \text { core. }}$ However, as the dose deposition occurs over a time $\Delta t \approx 1 \mathrm{~s}$, the measured temperature increase $\Delta T_{\text {core }}(\Delta t)$ will be lower due to the heat transfer to the surrounding materials during the irradiation. The heat loss correction $k_{\mathrm{ht}}$ is estimated in two ways as

$$
k_{\mathrm{ht}}=\frac{D_{\text {core }} / c_{p, \text { core }}}{\Delta T_{\text {core }}^{j}}, \quad \text { for } j=\text { ext, max }
$$

where $\Delta T_{\text {core }}^{\text {ext }}$ is the temperature change obtained through extrapolation of the post-irradiation temperature drift, and $\Delta T_{\text {core }}^{\max }$ is the peak of the average temperature change without extrapolation. $\Delta T_{\text {core }}^{\text {ext }}$ is closely related to the experimentally determined temperature rise, whereas $\Delta T_{\text {core }}^{\max }$ is used to set an upper limit for the heat transfer loss. The heat transfers are simulated with finite element method approaches (ANSYS Multiphysics v. 19, PA, USA). The heat transfer from the graphite core to the surroundings is investigated for the two doses $1.5 \mathrm{~Gy}$ and $3.0 \mathrm{~Gy}$, roughly corresponding to the dose at the entry channel and Bragg peak region, respectively. $k_{\mathrm{ht}}$ is estimated for the cases where the same dose is deposited over the times $\Delta t=\{1,2,5,10\} \mathrm{s}$ to simulate the effect of different dose rates.

The complex heating of the graphite core, where both the dose and spot widths increase with the depth, is in this work simplified by estimating an upper limit for $k_{\mathrm{ht}}$ in eq. (3): The energy deposition is set to be uniformly distributed in the graphite core over a time $\Delta t$ whereas no energy is deposited in the surroundings materials. Hence, the simulated heat transfer from the graphite core to the 172 solid water is to be overestimated relative to the experiments, where both the graphite core and 
surroundings were irradiated. The simulated temperature changes are eventually compared to the measured post-irradiation temperature drift.

The small graphite core pinned inside a solid water slab in figure 1 was implemented in ANSYS with the properties given in table 2. An adaptive mesh grid is generated in ANSYS, where the mesh is refined at the two pins holding the graphite core. The diameter of the circular contact surface between each solid water pin and the graphite core is set to be $1 \mathrm{~mm}$, which is larger than the actual size, and ensures a slight overestimation of the conductive heat transfer.

Table 2: Material properties used for simulating the heat transfers in ANSYS. The given values are estimated from Weast et al. (1988) unless otherwise stated.

\begin{tabular}{lcccc}
\hline Material & Mass density & $\begin{array}{c}\text { Specific heat } \\
\text { capacity } \\
{\left[\mathrm{J} \mathrm{kg} \mathrm{cm}^{-1} \mathrm{~K}^{-1}\right]}\end{array}$ & $\begin{array}{c}\text { Thermal } \\
\text { conductivity } \\
{\left[\mathrm{W} \mathrm{m}^{-1} \mathrm{~K}^{-1}\right]}\end{array}$ & Emissivity \\
\hline Graphite & 2.15 & 709 & 108 & $0.82^{a}$ \\
Solid Water HE & $1.032^{b}$ & 1100 & 0.90 & 0.95 \\
\hline
\end{tabular}

\subsection{Ionization quenching corrections}

The ionization quenching reduces the luminescence signal of the PSD $\left(L_{\mathrm{PSD}}\right)$ depending on the local ionization density in the PSD, and corrections relative to calorimetry requires other corrections than those based on e.g. ionometry. The relative quenching correction factor is experimentally determined as a function of the proton beam energy $E$ and the dose to the PSD ( $\left.D_{\mathrm{PSD}}\right)$ as

$$
k_{\text {quenching }}(E) \propto \frac{D_{\mathrm{PSD}}(E)}{L_{\mathrm{PSD}}(E)} \propto \frac{D_{\text {core }}(E) \cdot k_{\mathrm{vol}}^{\mathrm{MC}}(E)}{L_{\mathrm{PSD}}(E)} \propto \frac{\Delta T_{\text {core }}(E) \cdot k_{\mathrm{ht}}(E) \cdot k_{\mathrm{vol}}^{\mathrm{MC}}(E)}{L_{\mathrm{PSD}}(E)}
$$

using eqs. (1) and (2). $\Delta T_{\text {core }}$ and $L_{\mathrm{PSD}}$ are measured quantities, $k_{\mathrm{ht}}$ investigated with heat flow simulations, and $k_{\mathrm{vol}}^{\mathrm{MC}}$ derived from particle transport simulations. The experimentally based quenching correction factor is compared to theoretically calculated factors based on amorphous track structure approaches as presented in Christensen and Andersen (2018).

\section{Results and Discussion}

\subsection{Scintillator spot size measurements}

An example of a measurement with the PSD is shown in figure 2(a), where the PSD was placed approximately in the center of the spot-scanning field. The $5 \times 5$ proton spots gave rise to the 25 plateaus delineated with open circles, where the amplitude of the PSD signal varies with the distance from the spot to the PSD. A Gaussian function is fitted to each of the 5 scan lines, shown with a 196 solid line and filled area to assist the eye. The amplitude variation of the 5 Gaussians provides 
information about the proton spot size, and a single Gaussian is fitted to the maximum of each of them. The example in figure 2(a) gives a full width at half maximum (FWHM) of FWHM $=0.49 \mathrm{~s}$. The spot spread in time is converted into a distance given the measured $35.2 \mathrm{~ms}$ between spots separated $5 \mathrm{~mm}$ apart.

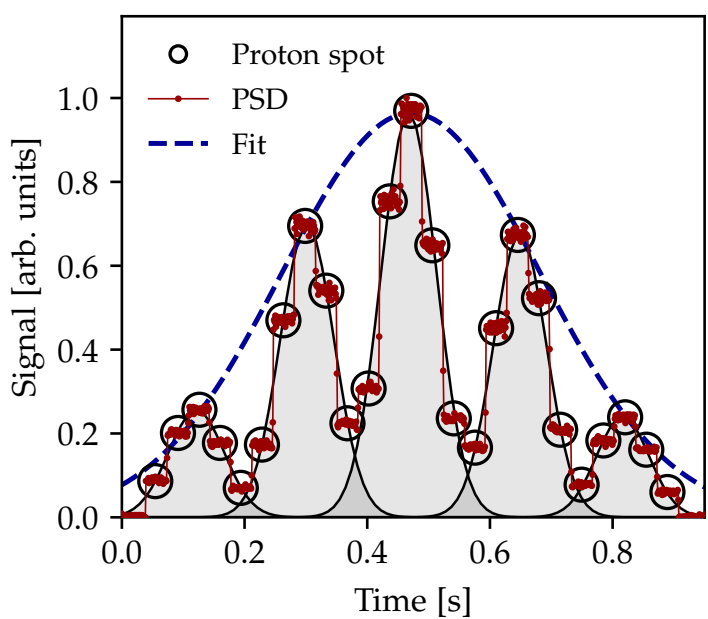

(a)

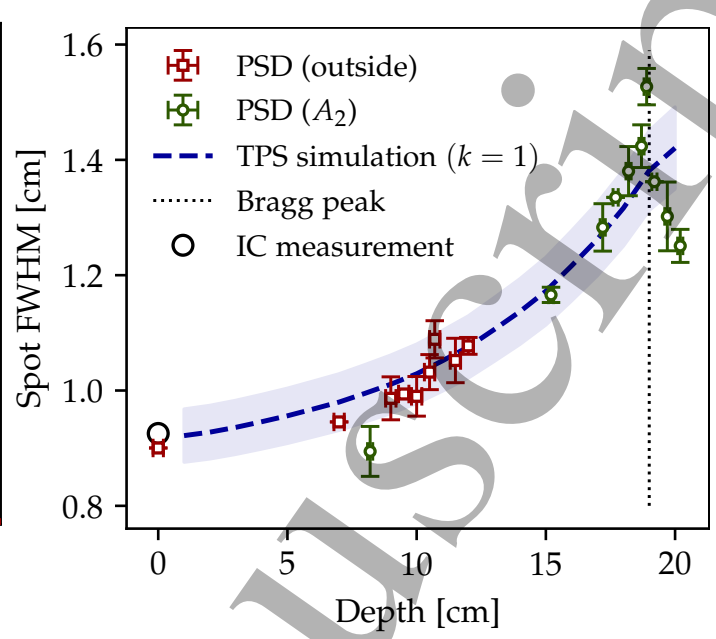

(b)

Figure 2: (a) The PSD signal as a function of time where the $5 \times 5$ spots are marked with circles. Gaussians are fitted to each of the five scan lines (filled gray) and a single Gaussian fitted (dashed line) to each of the five Gaussians. (b) The FWHM from the Gaussian fit in (a), converted into a length as described in section 3.1, for different depths of solid water as defined in figure 1 with $k=1$ uncertainties. The locations 'outside' and ' $A_{2}$ ' refer to the PSD position sketched in figure 1 . The TPS simulated spot widths are shown with a dashed line. The ionization chamber (IC) measurement of the proton spot is used for the Monte Carlo simulations.

The PSD measured FWHM of the spots is plotted in figure 2(b) for different depths $L$ of solid water slabs, where the $(k=1)$ errorbars are estimated from the fit to the 5 Gaussians, as shown in figure 2(a), given by the lmfit package for Python-3.8. The horizontal errorbars show the estimated $1 \mathrm{~mm}$ uncertainty of the positioning relative to the front of the solid water slabs. The treatment planning system (TPS; Eclipse, Varian, USA) simulated spot widths are in agreement with the measurements until the distal fall-off.

A similar disagreement between Monte Carlo simulated and measured spot widths was observed by Pedroni et al. (2005), which is attributed to the PSD measurement along the central beam axis, where the beam eventually narrows. If the middle proton spot-scanning line is not centred directly over the PSD, the spots are asymmetrically distributed around the center. Such an asymmetry is slightly visibly in figure 2(a) causing a small bias of the fitted Gaussian functions. The bias is not necessarily systematic but could explain the data outlier at $8 \mathrm{~cm}$ in figure 2(b).

Another, not quantified, uncertainty in the spot FWHM measurement is the presence of ionization quenching. The ionization density increases non-linearly with the depth, in particular at the central proton scan line. This in turn causes the central Gaussian in figure 2(a) to quench more with the depth than the neighbouring Gaussians, ultimately causing a too broad Gaussian to be fitted to all 5 scan lines giving an overestimation of the FWHM at the Bragg peak region. However, due to 
the small amount of quenching in proton beams, this effect is expected to be much smaller than the effect of a non-centred beam relative to the PSD. The proton spot size measurements with the PSDs provide information about the uniformity of the proton field and the temporal resolution of the spot depositions.

\subsection{Heat loss corrections}

The graphite calorimeter was evacuated to a $1 \mathrm{~Pa}$ air pressure which along with radiation induced temperature changes on a mK scale gives a negligible convection (Shipley and Duane, 1996; Ionita et al., 2010). The use of oversized contact surfaces between the graphite core and the solid water surroundings in the simulations ensures, combined with the lack of heat generation in the solid water, that both the conductive and radiative heat transfer losses from the graphite core to be overestimated. The ANSYS implementation of the graphite core inside the hollow solider water cylinder is shown in figure $3(\mathrm{a})$.

\subsubsection{Temperature simulations}

The dose to the entry channel and the Bragg peak region is roughly, approximated with 1.5 Gy and 3 Gy doses, respectively. The corresponding graphite temperature rise $\Delta T$, without any heat losses to the surroundings, is plotted in figure $3(\mathrm{~b})$ with horizontal dotted lines. The simulated average temperature increase of the graphite core as a function of time is plotted for both doses, where the energy is deposited in the graphite over $\Delta t=\{1,2,5,10\}$ s.

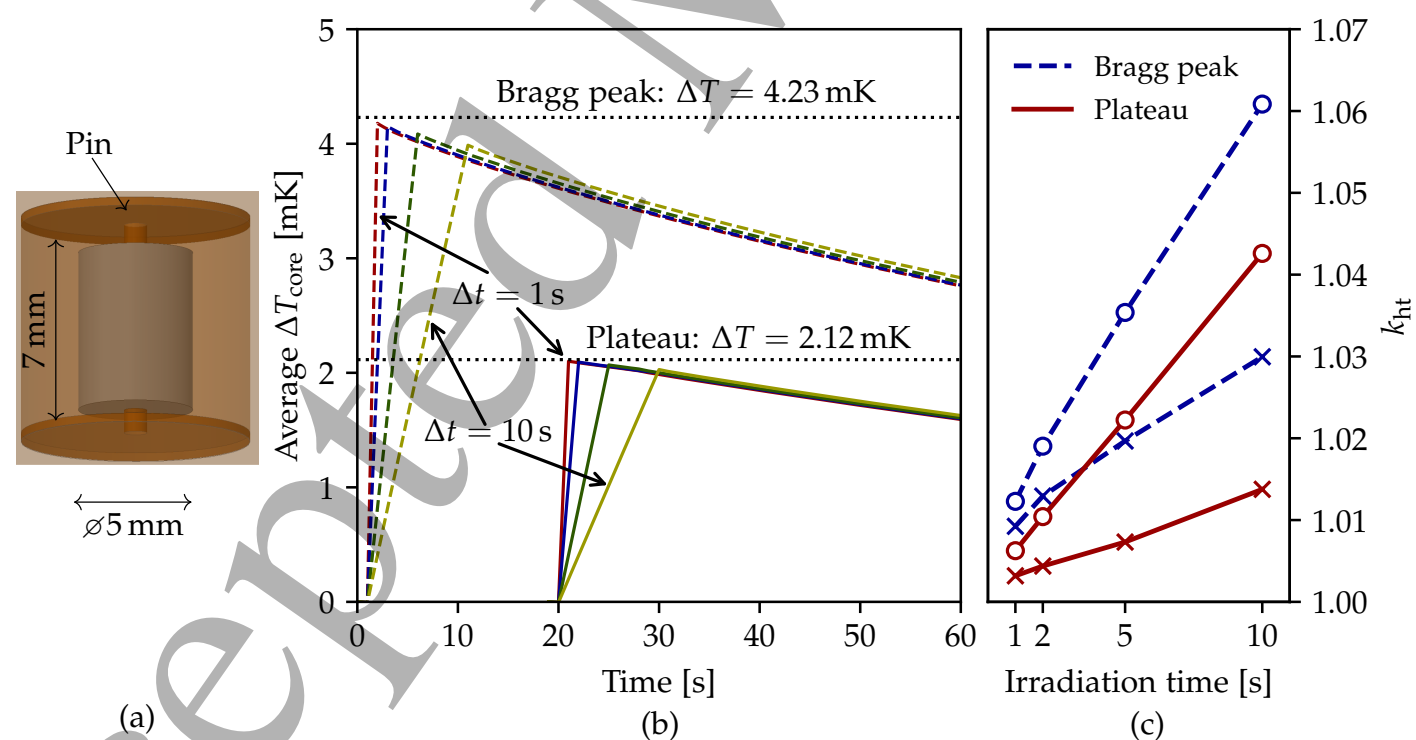

(b)

(c)

Figure 3: (a) ANSYS implementation of the small graphite core in the hollow solid water slab, confined by the two plastic pins. (b) Simulated average temperature change in the graphite core for different irradiation times $\Delta t=\{1,2,5,10\} \mathrm{s}$ at the plateau (solid lines) and Bragg peak (dashed lines) regions. The irradiations at the plateau region are shifted to start at $20 \mathrm{~s}$ only for visibility. (c) Estimated heat loss corrections $k_{\mathrm{ht}}$ for the 4 irradiation times in (b) based on eq. (3). Cross markers denote temperature changes obtained through the described extrapolation $\Delta T_{\text {core }}^{\text {ext }}$ whereas circle markers show the ones estimated through the $\Delta T_{\text {core }}^{\max }$ method. 
The heat transfer corrections $k_{\mathrm{ht}}$ are in figure 3(c) shown for the two methods outlined in eq. (3). The heat transfer correction based on the extrapolation of the post-irradiation temperature drift $\left(\Delta T_{\text {core }}^{\text {ext }}\right.$ cross markers), i.e. similar to the experimental approach, gives $k_{\mathrm{ht}}=1.003$ and $k_{\mathrm{ht}}=1.009$ at the plateau and Bragg peak region, respectively. The correction based on the peak of the average temperature rise ( $\Delta T_{\text {core }}^{\max }$; circle markers), gives upper estimates of the heat transfer corrections as $k_{\mathrm{ht}}=1.006$ at the plateau region and $k_{\mathrm{ht}}=1.012$ at the Bragg peak region when the field is delivered in $1 \mathrm{~s}$. However, the results indicate that heat transfer corrections are important for the calorimeter for lower dose-rates. The temperature simulations furthermore indicate an upper limit for the post-irradiation temperature drift from the graphite core to the surroundings to be of the order of $25 \mu \mathrm{K} \mathrm{s}^{-1}$ after the maximum temperature has been reached.

\subsubsection{Temperature measurements}

Two temperature measurements of the small graphite core pre- and post-irradiation are shown in figure 4(a) and (b). The temperature of the graphite core is drifting before and after each irradiations, where linear functions are fitted to the data in each case. The temperature change is obtained from extrapolating the pre- and post-irradiation temperatures shown with yertical, dotted lines.

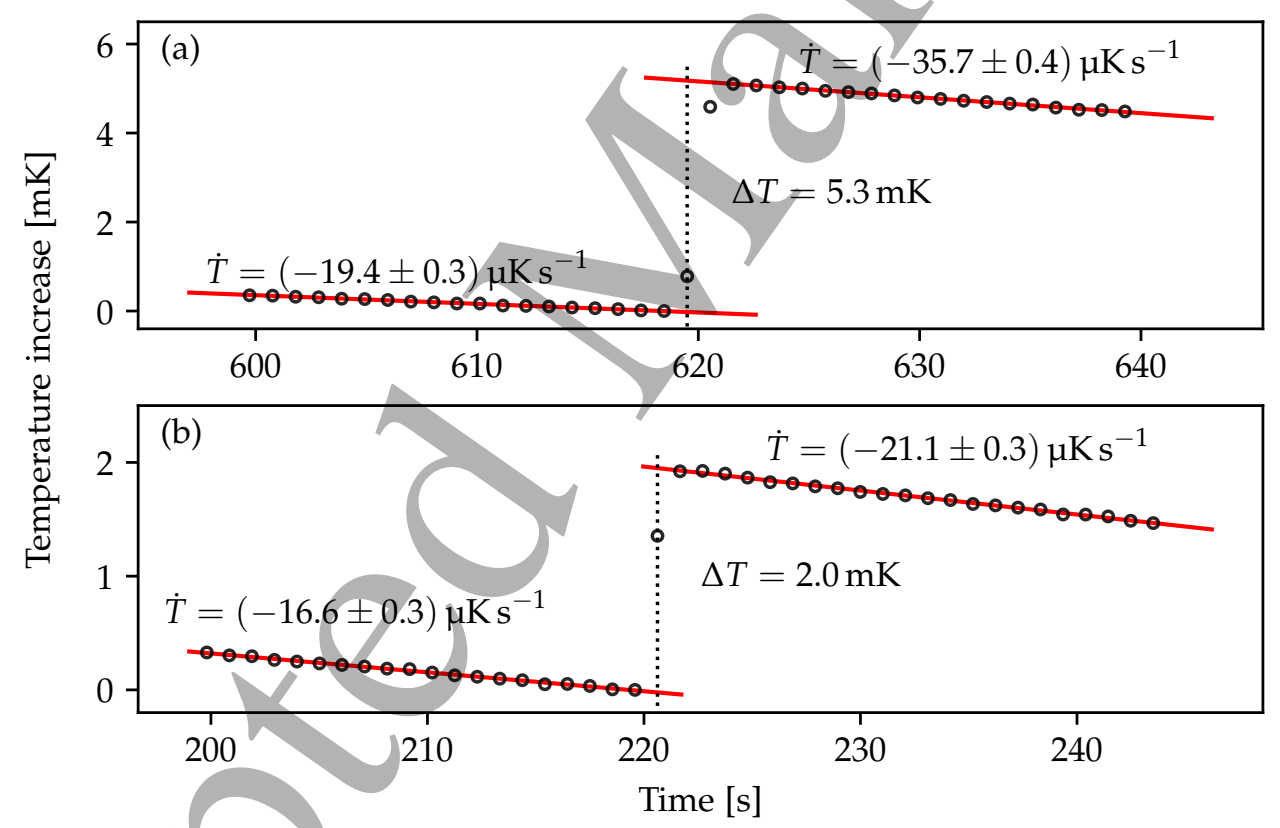

Figure 4: The temperature response of the small graphite core to two different doses deposited in about $0.9 \mathrm{~s}$. The graphite core acquired a uniform temperature in $\approx 2 \mathrm{~s}$ in both cases. The temperature changes extrapolated at the dotted lines are given in each figure along with the preand post-irradiation temperature drifts. (a) is measured for a solid water thickness of $10.5 \mathrm{~cm}$ and (b) $2 \mathrm{~cm}$.

The measured post-irradiation drifts within $20 \mathrm{~s}$, subtracted the pre-irradiation drift, is around $4.5 \mu \mathrm{K} \mathrm{s}^{-1}$ at the entry channel and $16.3 \mu \mathrm{K} \mathrm{s}^{-1}$ at the Bragg peak. The measured drift rate is thus much smaller than the simulated post-drift rate around $25 \mu \mathrm{K} \mathrm{s}^{-1}$, in agreement with the simulations 254 acting as an upper limit for the heat losses. The ratio of the post-irradiation temperature drift to 
the temperature increase amounts to $0.2 \% \mathrm{~s}^{-1}$ at the entry channel and $0.3 \% \mathrm{~s}^{-1}$ at the Bragg peak, experimentally indicating a negligible variation of the heat transfer loss for a $1 \mathrm{~s}$ irradiation.

\subsection{Calorimeter response}

The measured temperature changes in the small graphite core are shown in figure 5 as a function of depth. Each data point is the result of 5 repeated measurements with a typical relative standard deviation less than $0.5 \%(10 \mu \mathrm{K})$. The Geant4 simulated dose to the small graphite core for a broad range of solid water thicknesses gave a quasi-continuous function consistent with the calorimeter measurements ( $<2.8 \%$ deviation below the distal $80 \%$ dose point) as depicted in figure 5 .

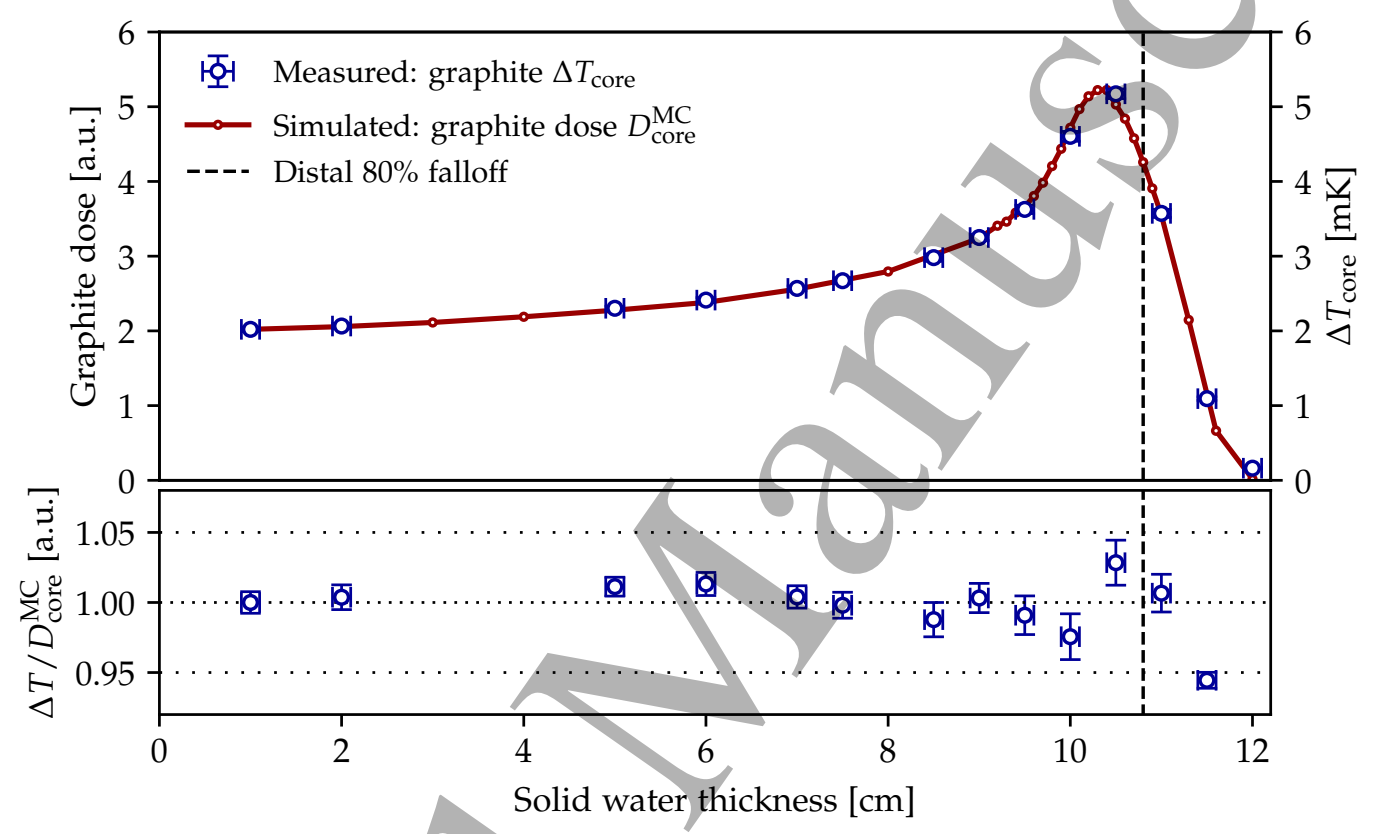

Figure 5: The Geant4 simulated dose $D_{\text {core }}^{\mathrm{MC}}$ to the small graphite core along with the measured temperature change $\Delta T_{\text {core }}$ for the same graphite core for different thicknesses $L$ of solid water slabs.

The consistency between the validated Monte Carlo model simulation of the dose and the measured temperature increase in the graphite core indicates, that the graphite calorimeter temperature response is approximately proportional to the absorbed dose. Hence, the result indicates a constant heat loss correction $k_{\mathrm{ht}}$ along the central beam axis. This is in agreement with the assessment of the heat transfers at the plateau and Bragg peak regions, using the simulations in section 3.2.1 and measurements in section 3.2.2. Thus, the heat loss correction $k_{\mathrm{ht}}$ is neglected for the calculation of $k_{\text {quenching }}$ as the relative corrections vary less than $1 \%$. However, a $1 \%$ relative uncertainty of $k_{\mathrm{ht}}$ is added to the total uncertainty of $k_{\text {quenching, }}$ corresponding to the maximum variation along the central beam axis. 


\subsubsection{Scintillator quenching corrections}

The Monte Carlo model enables the calculation of volume averaging correction factors in eq. (2) along with the $\mathrm{LET}_{\Phi}$ in the PSD, which typically is used as a quenching predictor for scintillators. The measured temperature change of the graphite core corrected for volume averaging, which is proportional to the dose deposited in the PSD cf. eq. (4), is shown in figure 6 next to the measured PSD response and the simulated $\mathrm{LET}_{\Phi}$ distribution. The annotated $L_{\mathrm{PSD}}$ data point at $10.7 \mathrm{~cm}$ ( $40 \mathrm{MeV} \mathrm{cm}^{2} \mathrm{~g}^{-1}$ ) is without a corresponding temperature measurement of the graphite core, i.e.

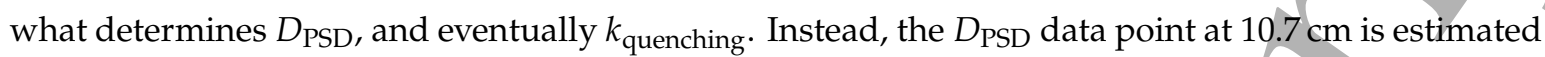
through linear interpolation of the two neighbouring data points.

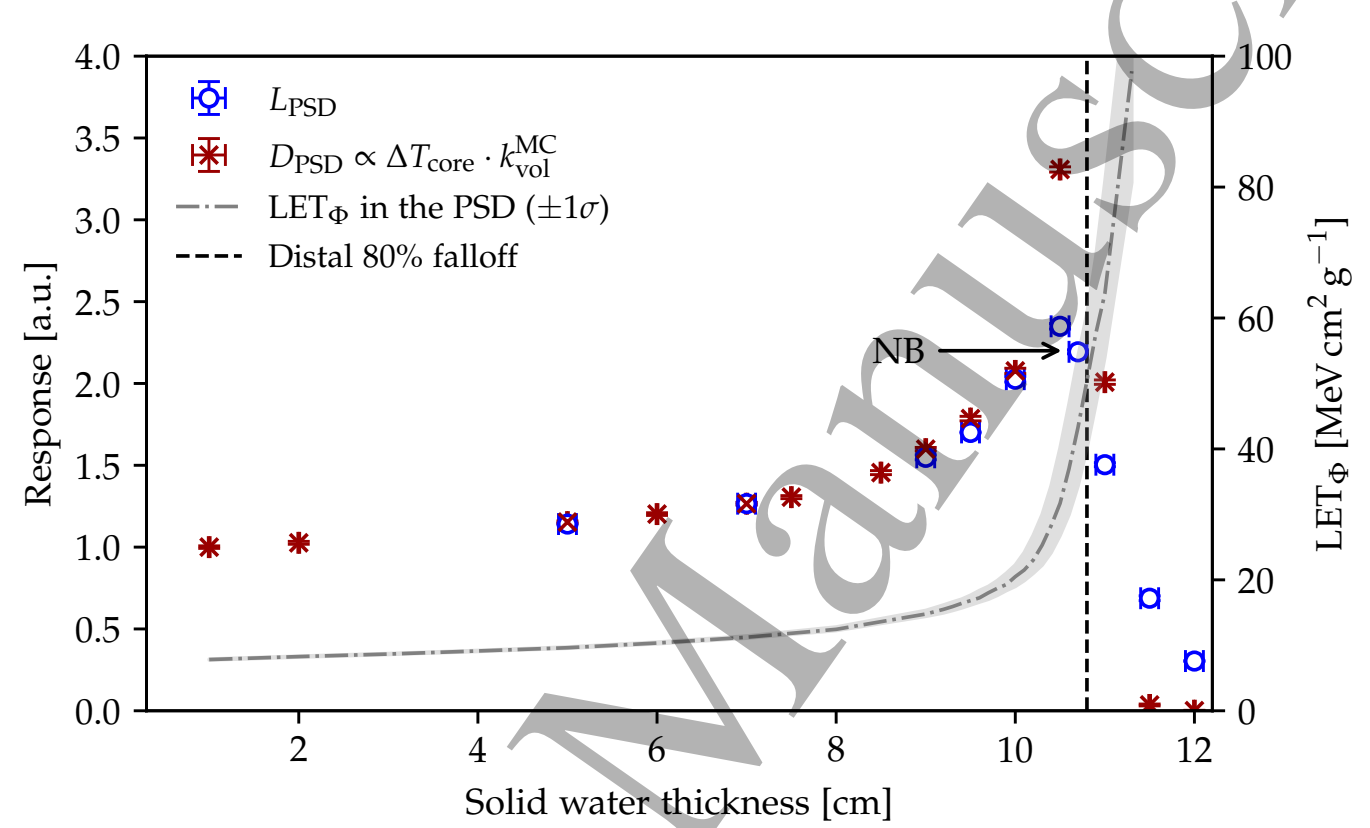

Figure 6: The quenched PSD luminescence signal $\left(L_{\mathrm{PSD}}\right)$ relative to the dose to the PSD $\left(D_{\mathrm{PSD}}\right)$ calculated as the temperature increase of the graphite core adjusted by the volume averaging correction. The $\operatorname{LET}_{\Phi}$ with estimated ( $k=1$, type B) uncertainties are plotted for reference. The NB annotation shows the $L_{\mathrm{PSD}}$ measurement without a corresponding $D_{\mathrm{PSD}}$ measurement.

The quenching correction factor based on eq. (4) is shown in figure 7 as a function of the $\mathrm{LET}_{\Phi}$. The measured quenching correction factors are in the figure compared to theoretical quenching corrections calculated with the open-source code ExcitonQuenching (Christensen and Andersen, 2018), relying on amorphous track structure models and scintillator material properties, plotted with a dashed line. The data point at $40 \mathrm{MeV} \mathrm{cm}^{2} \mathrm{~g}^{-1}$ is omitted in the linear fit as the corresponding temperature change of the graphite core was not measured (see figure 6), but approximated through a linear interpolation, which typically is inadequate at such steep dose gradients.

The slope of the linear fit in figure 7 , denoted $k B$, is a measure of the amount of quenching in PSDs as defined in the Birks formalism (Birks, 1951). The value of $k B=(11.2 \pm 0.4) \mathrm{mg} \mathrm{MeV}^{-1} \mathrm{~cm}^{-2}$ for the BCF-60 PSD is in agreement with the $k B=(10.9 \pm 0.4) \mathrm{mg} \mathrm{MeV}^{-1} \mathrm{~cm}^{-2}$ value obtained relative to ionometry derived in Christensen et al. (2019) and the applied theoretical model. The consistency between theory and measurements indicates that the graphite calorimeter is applicable 


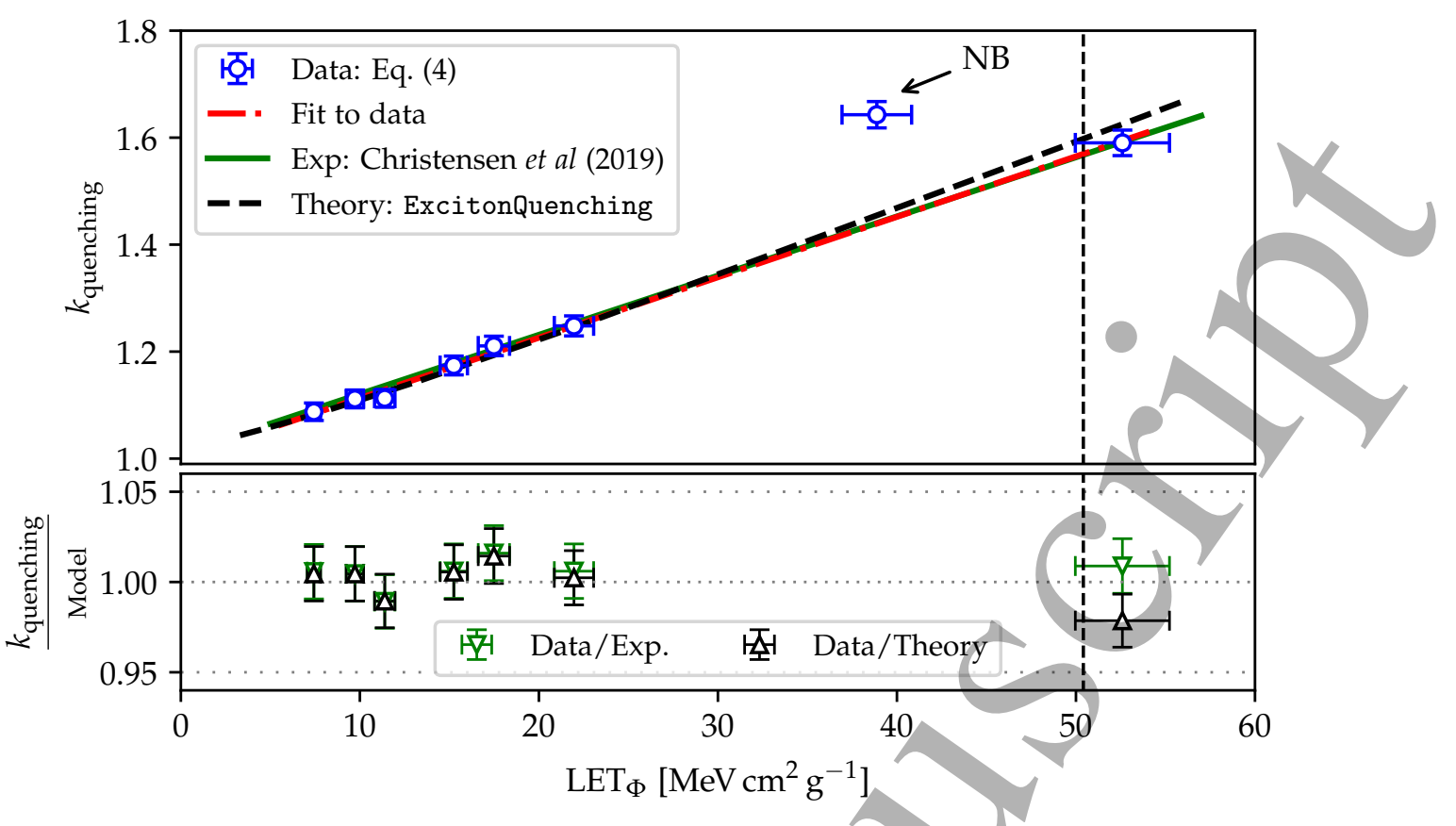

Figure 7: The quenching correction factor $k_{\text {quenching }}$ obtained from eq. (4) as a function of $\mathrm{LET}_{\Phi}$. The theoretical correction factor (dashed line) is calculated with the algorithm ExcitonQuenching along with an experimental value (solid line) derived relative to ionometry. The NB-annotated point, as explained in the text, is neither used for the fit (dot-dashed line) or shown in the ratio of the data to the experimental and theoretical values in the lower subfigure.

for the measurement of quenching correction in PSDs.

\subsubsection{Uncertainty components} $\mathrm{LET}_{\Phi}$ and $k_{\text {quenching }}$ uncertainties in table 3.

\section{Quenching correction challenges}

The combined 'Type $\mathrm{A}^{\prime}$ and 'Type $\mathrm{B}$ ' uncertainty of the quenching correction factor $k_{\text {quenching, }}$ depending on the parameters given in eq. (4), is given in table 3. The Monte Carlo simulated $\mathrm{LET}_{\Phi}$ distribution is known to depend strongly on the scoring method (Grzanka et al., 2018), an in particular on the inclusion of secondary particles. A small offset in the scoring at the Bragg peak region will thus greatly affect the scored LET. The possible systematic misalignment is included in the estimation of the 'Type B' uncertainty, by investigating the LET variation given a scoring volume shift of $1 \mathrm{~mm}$ in either direction along the central beam axis. A similar approach is used to estimate the 'Type B' uncertainty of the other Monte Carlo quantities.

The quenching parameter $k B$ is estimated from a linear fit for $k_{\text {quenching }}$ versus $\mathrm{LET}_{\Phi}$ as given in figure 7. The uncertainty of $k B$ is estimated with the lmfit package in Python-3.8 using the given

The present work corrects the ionization quenching in scintillators relative to absorbed dose calorimetry, in contrast to more common approaches where the response is corrected theoretically or exper310 imentally relative to an ionization chamber (IC). ICs are generally only applicable for quenching 
Table 3: Uncertainty components given as the relative uncertainty expressed as a percentage. The Monte Carlo 'Type B' uncertainties are estimated at the Bragg peak for a $1 \mathrm{~mm}$ offset as discussed in the text.

\begin{tabular}{lcc}
\hline Source of uncertainty component & Type A [\%] & Type B [\%] \\
\hline LET $_{\Phi}$ scoring & 0.1 & 5.0 \\
Graphite temperature $\Delta T_{\text {core }}$ & $<0.2$ & 0.2 \\
PSD signal $L_{\mathrm{PSD}}$ & 1.0 & $<0.1$ \\
Volume averaging $k_{\mathrm{vol}}^{\mathrm{MC}}$ & 0.1 & 1.5 \\
Heat transfer $k_{\mathrm{ht}}^{\dagger}$ & 0.5 & $<1.0$ \\
\hline Combined $k_{\text {quenching }}($ eq. $(4))$ & \multicolumn{2}{c}{2.1} \\
\hline
\end{tabular}

${ }^{+} k_{\mathrm{ht}}$ is neglected in the calculation of $k_{\text {quenching }}$ but included in its uncertainty.

references in cases where the initial and general recombination can be neglected, i.e. not for FLASH or other complex beams. The recombination in ionization chamber irradiated with scanning ion beams is, nonetheless, generally left uncorrected along the central beam axis, including the Bragg peak region. The measured under-response hence leads to an underestimated quenching parameter determination.

Theoretically based approaches, that be amorphous track structure based as presented in Christensen and Andersen (2018), or a mathematical model based on the Birks model as in Kelleter and Jolly (2020), rely on the LET distribution. The LET scoring furthermore depends on the choice of implementation and beam parameters, in particular around the distal edge where the quenching is large and the LET scoring uncertain.

The use of the new graphite calorimeter for quenching corrections avoids both the underresponse of the reference detector due to ionic recombination and reduces the relative positional misalignments as the PSD is placed inside the graphite core. The thicker and denser graphite core, on the other hand, causes a larger signal averaging relative to the smaller PSD which is problematic at steep dose gradients. Whilst the heat losses of the graphite core could lead to an underestimation of the temperature increase, and hence an underestimation of the quenching, the heat loss was here demonstrated to be negligible for the high intensity scanning proton beam.

The graphite calorimeter will thus be applicable for detector characterization in complex beams, e.g. the plateau region of a heavy ion beam or a FLASH intensity beam where detectors as ionization chambers are insufficient.

\section{Conclusion}

A graphite calorimeter for dosimetry in high-intensity beams is presented. The calorimeter incorporates a plastic scintillator which with its prompt response facilitates a measurement of each spot in a spot-scanning field. The calorimeter was tested in a $170 \mathrm{MeV}$ spot-scanning proton beam where the plastic scintillator correctly identified each spot in the field as well as the proton spot size along the 336 central beam axis. The response of the graphite calorimeter is reproducible within $0.5 \%$ correspond- 
ing to $\mu \mathrm{K}$ temperature variations of the measurements. An upper estimate of the heat transfer loss during the $\approx 0.9 \mathrm{~s}$ irradiation was found to be $0.3 \%$ at the entry channel and $0.9 \%$ at the Bragg peak based on simulations, consistent with the extrapolated measurements of $0.2 \% \mathrm{~s}^{-1}$ and $0.3 \% \mathrm{~s}^{1}$, respectively. The negligible variation of the heat transfer correction is furthermore supported by the measured temperature increase of the graphite core and Monte Carlo simulations of the dose deposition, which indicates a proportionality between the dose deposition and the temperature rise.

Finally, the new approach of correcting the ionization quenched scintillator response relative to the temperature increase of the graphite core was validated against more common approaches. The quenching correction parameter relative to calorimetry was found to be $k B=(11.2 \pm 0.4) \mathrm{mg} \mathrm{MeV}^{-1} \mathrm{~cm}^{-2}$ for the BCF-60 scintillator, in agreement with theoretically and experimentally based literature values.

\section{Acknowledgements}

The authors thank Peter Sandegaard Skyt and Christian Skou Søndergaard, Danish Centre for Particle Therapy, for measurement assistance and for providing the simulated proton spot sizes. The work was supported by the Danish Council for Independent Research (grant FTP, DFF 4184-00151), the Danish Cancer Society, and the Danish Comprehensive Cancer Center (DCCC RT).

\section{References}

Agostinelli, S., J. Allison, K. al Amako, J. Apostolakis, H. Araujo, P. Arce, M. Asai, D. Axen, S. Banerjee, G. Barrand, and Others (2003). GEANT4-a simulation toolkit. Nuclear instruments and methods in physics research section A: Accelerators, Spectrometers, Detectors and Associated Equipment 506(3), 250-303.

Archambault, L., J. C. Polf, L. Beaulieu, and S. Beddar (2008). Characterizing the response of miniature scintillation detectors when irradiated with proton beams. Physics in Medicine and Biology 53(7), 1865.

Beierholm, A. R., C. F. Behrens, and C. E. Andersen (2014). Dosimetric characterization of the exradin w1 plastic scintillator detector through comparison with an in-house developed scintillator system. Radiation measurements 69, 50-56.

Beierholm, A. R., R. O. Ottosson, L. R. Lindvold, C. F. Behrens, and C. E. Andersen (2011). Characterizing a pulse-resolved dosimetry system for complex radiotherapy beams using organic scintillators. Physics in Medicine and Biology 56(10), 3033.

Birks, J. B. (1951). Scintillation from organic crystals: Specific flourescence and relative response to different radiation. Proceedings of the Physical Society Section A 64(10), 874-877.

Burns, D. (2012). An analysis of existing data for $w_{\mathrm{air}}, i_{\mathrm{c}}$ and the product $w_{\mathrm{air}} s_{\mathrm{c}, \text { air }}$. Metrologia 49 , 507-512. 
Christensen, J. B., E. Almhagen, L. Stolarczyk, M. Liszka, G. G. Hernandez, N. Bassler, O. Nørrevang, and A. Vestergaard (2020). Mapping initial and general recombination in scanning proton pencil beams. Physics in Medicine and Biology.

Christensen, J. B., E. Almhagen, L. Stolarczyk, A. Vestergaard, N. Bassler, and C. E. Andersen (2019). Ionization quenching in scintillators used for dosimetry of mixed particle fields. Physics in Medicine and Biology 64(9), 095018.

Christensen, J. B. and C. E. Andersen (2018). Relating ionization quenching in organic plastic scintillators to basic material properties by modelling excitation density transport and amorphous track structure during proton irradiation. Physics in Medicine and Biology 63(19), 195010.

Christensen, J. B., G. V. Santurio, A. Vestergaard, S. Korreman, and C. E. Andersen (2020). Graphite calorimetry for quantifying ionization quenching in plastic scintillators in proton dosimetry. $R a$ diation Measurements 132, 106277.

Cortés-Giraldo, M. and A. Carabe (2015). A critical study of different Monte Carlo scoring methods of dose average linear-energy-transfer maps calculated in voxelized geometries irradiated with clinical proton beams. Physics in Medicine and Biology 60(7), 2645.

Duane, S., M. Aldehaybes, M. Bailey, N. D. Lee, C. G. Thomas, and H. Palmans (2012). An absorbed dose calorimeter for IMRT dosimetry. Metrologia 49(5).

Grzanka, L., O. Ardenfors, and N. Bassler (2018). Monte Carlo simulations of spatial LET distributions in clinical proton beams. Radiation protection dosimetry 180(1-4), 296-299.

Ionita, C., D. Radu, and I. Astefanoaei (2010). Radiative Heat loss correction for 3-body graphite calorimeters. Acta Physica Polonica A 118(4), 550-562.

Kelleter, L. and S. Jolly (2020). A mathematical expression for depth-light curves of therapeutic proton beams in a quenching scintillator. Medical Physics.

Kry, S. F., P. Alvarez, J. E. Cygler, L. A. DeWerd, R. M. Howell, S. Meeks, J. O'Daniel, C. Reft, G. Sawakuchi, E. G. Yukihara, et al. (2020). AAPM TG 191: Clinical use of luminescent dosimeters: TLDs and OSLDs. Medical Physics 47(2)

Kumar, S., J. D. Fenwick, T. S. Underwood, D. D. Deshpande, A. J. Scott, and A. E. Nahum (2015). 398 Breakdown of Bragg-Gray behaviour for low-density detectors under electronic disequilibrium conditions in small megavoltage photon fields. Physics in Medicine and Biology 60(20), 8187.

400 Lye, J., P. Harty, D. Butler, J. Crosbie, J. Livingstone, C. Poole, G. Ramanathan, T. Wright, and A. Stevenson (2016). Absolute dosimetry on a dynamically scanned sample for synchrotron radiotherapy using graphite calorimetry and ionization chambers. Physics in Medicine and Biology 61(11), 4201. 
Marsolat, F., L. De Marzi, A. Patriarca, C. Nauraye, C. Moignier, M. Pomorski, F. Moignau, S. Heinrich, D. Tromson, and A. Mazal (2016). Dosimetric characteristics of four PTW microdiamond detectors in high-energy proton beams. Physics in Medicine and Biology 61(17), 6413.

McEwen, M. R. and S. Duane (2000). A portable calorimeter for measuring absorbed dose in the radiotherapy clinic. Physics in Medicine and Biology 45(12), 3675-3691.

Pedroni, E., S. Scheib, T. Böhringer, A. Coray, M. Grossmann, S. Lin, and A. Lomax (2005). Experimental characterization and physical modelling of the dose distribution of scanned proton pencil beams. Physics in Medicine and Biology 50(3), 541-561.

Perl, J., J. Shin, J. Schümann, B. Faddegon, and H. Paganetti (2012). TOPAS: an innovative proton Monte Carlo platform for research and clinical applications. Medical physies 39(11), 6818-6837.

Picard, S., D. T. Burns, and P. Roger (2009). Construction of an absorbed-dose graphite calorimeter. Technical report, BIPM, Sèvres Cedex.

Pinto, M., M. Pimpinella, M. Quini, M. D’Arienzo, I. Astefanoaei, S. Loreti, and A. Guerra (2016). A graphite calorimeter for absolute measurements of absorbed dose to water: application in medium-energy x-ray filtered beams. Physics in Medicine and Biology 61(4), 1738.

Renaud, J., H. Palmans, A. Sarfehnia, and J. Seuntjens (2020). Absorbed dose calorimetry. Physics in Medicine and Biology 65(5).

Renaud, J., A. Sarfehnia, J. Bancheri, and J. Seuntjens (2018). Aerrow: A probe-format graphite calorimeter for absolute dosimetry of high-energy photon beams in the clinical environment. Medical Physics 45(1), 414-428.

Santurio, G. V. and C. E. Andersen (2019). Quantifying the ionization quenching effect in organic plastic scintillators used in MV photon dosimetry. Radiation Measurements 129, 106200.

Santurio, G. V., M. Pinto, and C. E. Andersen (2020). Evaluation of the ionization quenching effect in an organic plastic scintillator using $\mathrm{kV}$ x-rays and a modified Birks model with explicit account of secondary electrons. Radiation Measurements 131, 10622.

Sechopoulos, I., D. W. Rogers, M. Bazalova-Carter, W. E. Bolch, E. C. Heath, M. F. McNitt-Gray, J. Sempau, and J. F. Williamson (2017). RECORDS: improved Reporting of montE CarlO RaDiation transport Studies: report of the AAPM research committee task group 268. Medical Physics 45(1)

432 Shipley, D. R. and S. Duane (1996). Heat loss mechanisms in a measurement of specific heat capacity of graphite. CIRA-EXT 0039.

Torrisi, L. (2000). Plastic scintillator investigations for relative dosimetry in proton-therapy. $\mathrm{Nu}$ clear Instruments and Methods in Physics Research Section B: Beam Interactions with Materials and Atoms 170(3-4), 523-530. 
van de Water, S., S. Safai, J. M. Schippers, D. C. Weber, and A. J. Lomax (2019). Towards flash proton

438

440

442

444

Weast, R. C., M. J. Astle, and W. H. Beyer (1988). CRC handbook of chemistry and physics. CRC therapy: the impact of treatment planning and machine characteristics on achievable dose rates. Acta Oncologica 58(10), 1463-1469.

Waldeland, E., E. O. Hole, E. Sagstuen, and E. Malinen (2010). The energy dependence of lithium formate and alanine EPR dosimeters for medium energy x-rays. Medical Physics 37, 3569-3575.

Wang, L., L. Perles, L. Archambault, N. Sahoo, D. Mirkovic, and S. Beddar (2012). Determination of the quenching correction factors for plastic scintillation detectors in therapeutic high-energy proton beams. Physics in Medicine and Biology 57(23), 7767.

press Boca Raton, FL. 